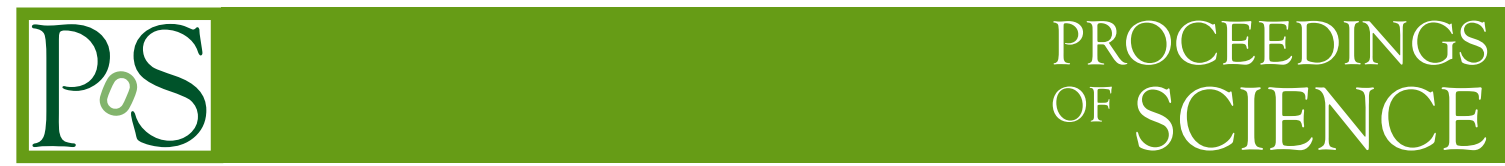

\title{
Searches for charged Higgs bosons at CMS
}

\author{
Santeri Laurila*t \\ Helsinki Institute of Physics \\ E-mail: santeri.laurilaecern.ch
}

\begin{abstract}
An overview of the results on the charged Higgs boson searches by the CMS Collaboration is presented. As different models with extended Higgs sectors predict different production and decay modes for these particles, the CMS search program for charged Higgs bosons covers a variety of final states targeting different models. The results of searches for charged Higgs bosons decaying into $c \bar{s}, c \bar{b}, t \bar{b}, \tau^{+} v_{\tau}, \mathrm{W}^{+} \mathrm{Z}$ (or charge-conjugate final states) and for doubly-charged Higgs bosons decaying into $\mathrm{W}^{ \pm} \mathrm{W}^{ \pm}$are summarized. The observed limits agree with the standard model predictions.
\end{abstract}

The 39th International Conference on High Energy Physics (ICHEP2018)

4-11 July, 2018

Seoul, Korea

*Speaker.

${ }^{\dagger}$ On behalf of the CMS Collaboration. 


\section{Introduction}

Several extensions of the standard model predict a complex Higgs sector with several Higgs fields, yielding a spectrum of Higgs bosons with different masses, charges and other properties. The CMS Collaboration has a wide search program for charged Higgs bosons $\left(\mathrm{H}^{ \pm}\right)$, with different search channels motivated by different models.

Two-Higgs-doublet models (2HDMs) predict five Higgs bosons, two of which are charged [1]. In $2 \mathrm{HDMs}$, the dominant $\mathrm{H}^{+}$decay modes are typically $\tau^{+} v_{\tau}$ and $\mathrm{t} \overline{\mathrm{b}}$, or $\mathrm{c} \overline{\mathrm{b}}$ and $\mathrm{c} \overline{\mathrm{s}}$, depending on how the two Higgs doublets couple to fermions. (Charge-conjugate processes with $\mathrm{H}^{-}$are always implied.) The minimal supersymmetric standard model is a special case of 2HDM [2].

The $\mathrm{H}^{ \pm}$are also predicted by more complex models, such as triplet models [3, 4]. The phenomenology of the triplet models is different from $2 \mathrm{HDMs}$, as $\mathrm{H}^{ \pm}$couples to vector bosons at tree level and double-charged Higgs bosons $\left(\mathrm{H}^{++}\right)$are introduced.

In the following, the CMS search results for charged Higgs bosons decaying into $c \bar{s}[5], c \bar{b}[6]$, $\mathrm{t} \overline{\mathrm{b}}[7], \tau^{+} v_{\tau}[8,7,9,10]$, and $\mathrm{W}^{+} \mathrm{Z}[11,12]$ are summarized. The results of a search for doublycharged Higgs bosons decaying into $\mathrm{W}^{+} \mathrm{W}^{+}[13]$ are also presented.

\section{The CMS detector}

The central feature of the CMS apparatus is a superconducting solenoid of $6 \mathrm{~m}$ internal diameter, providing a magnetic field of $3.8 \mathrm{~T}$. Within the solenoid volume are a silicon pixel and strip tracker, a lead tungstate crystal electromagnetic calorimeter, and a brass and scintillator hadron calorimeter, each composed of a barrel and two endcap sections. Forward calorimeters extend the pseudorapidity coverage provided by the barrel and endcap detectors. Muons are detected in gasionization chambers embedded in the steel flux-return yoke outside the solenoid. Events of interest are selected using a two-tiered trigger system. A more detailed description of the CMS detector, together with a definition of the coordinate system used and the relevant kinematic variables, can be found in Ref. [14].
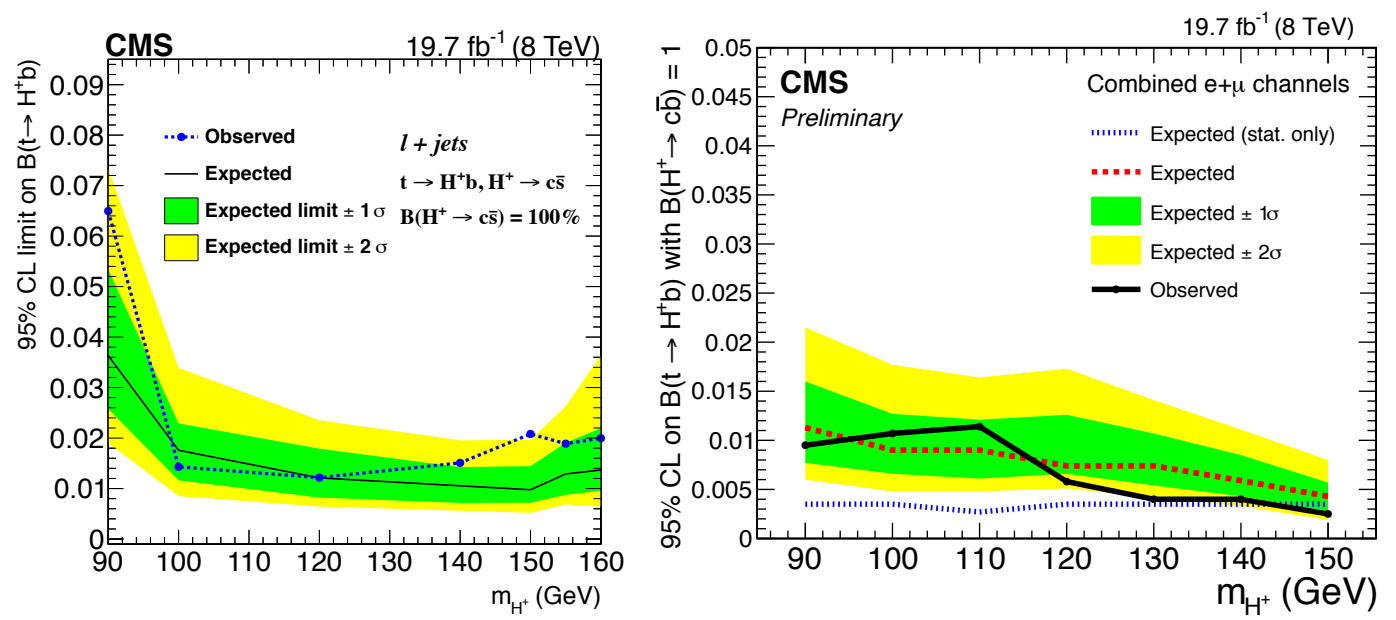

Figure 1: The observed (solid black lines) and expected (dashed lines) 95\% confidence level (CL) upper limits on the branching fraction of $\mathrm{t} \rightarrow \mathrm{bH}^{ \pm}$, with $\mathrm{H}^{+}$decaying exclusively into $c \bar{s}$ (left) [5] or c $\bar{b}$ (right) [6]. 


\section{Results}

A set of analyses targeting $\mathrm{H}^{ \pm}$as predicted by the $2 \mathrm{HDM}$ was performed based on $19.7 \mathrm{fb}^{-1}$ of data collected at center-of-mass energy of $\sqrt{s}=8 \mathrm{TeV}$. The results of searches for $\mathrm{H}^{+} \rightarrow \mathrm{cs}$ [5] and $\mathrm{H}^{+} \rightarrow \mathrm{cb}[6]$ are summarized in Fig. 1. Also $\mathrm{H}^{+} \rightarrow \mathrm{tb}$ (Fig. 2, left) and $\mathrm{H}^{+} \rightarrow \tau^{+} v_{\tau}$ channels were studied, covering $\mathrm{H}^{ \pm}$mass range up to $600 \mathrm{GeV}$ [7]. The $\tau^{+} v_{\tau}$ channel was revisited at $\sqrt{s}=13 \mathrm{TeV}$ in 2016, extending the search range to $3 \mathrm{TeV}$ as shown in Fig 2 (right) [9]. ${ }^{1}$
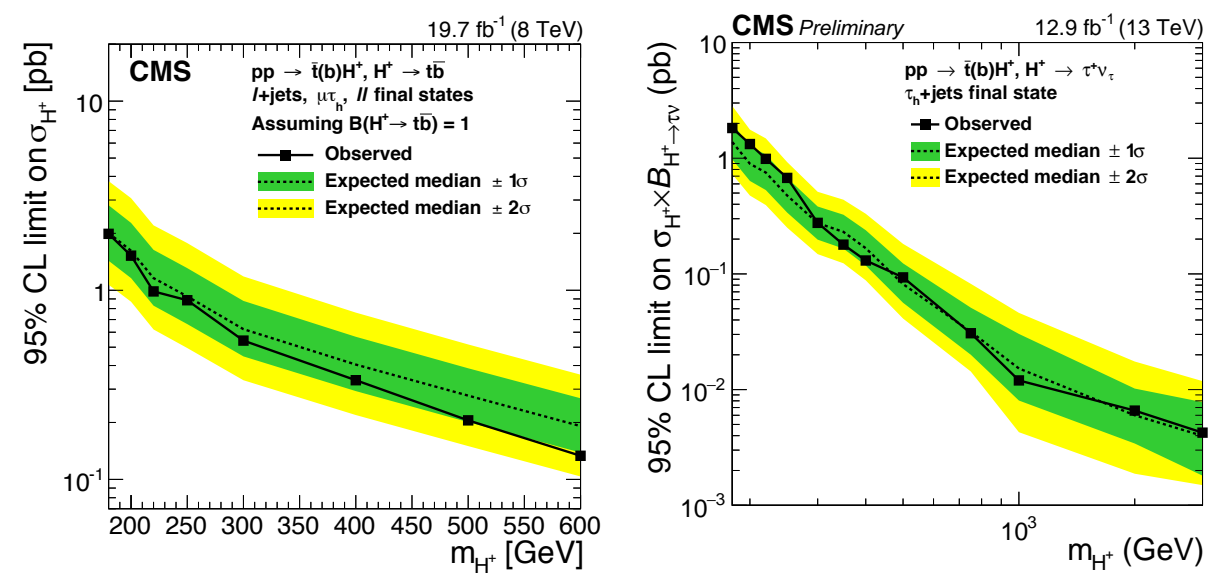

Figure 2: The observed (solid black lines) and expected (dashed lines) 95\% CL upper limits on the $\mathrm{H}^{ \pm}$ production cross section, assuming $\mathrm{H}^{+}$decaying exclusively into tb (left) [7] or $\tau^{+} v_{\tau}$ (right) [9].

The $\mathrm{H}^{ \pm}$and $\mathrm{H}^{ \pm \pm}$produced in vector boson fusion and decaying as $\mathrm{H}^{+} \rightarrow \mathrm{W}^{+} \mathrm{Z}$ [12] and $\mathrm{H}^{++} \rightarrow \mathrm{W}^{+} \mathrm{W}^{+}$[13], as predicted by triplet models, were searched for using $35.9 \mathrm{fb}^{-1}$ of data collected at center-of-mass energy of $\sqrt{s}=13 \mathrm{TeV}$. These results are summarized in Fig. 3.
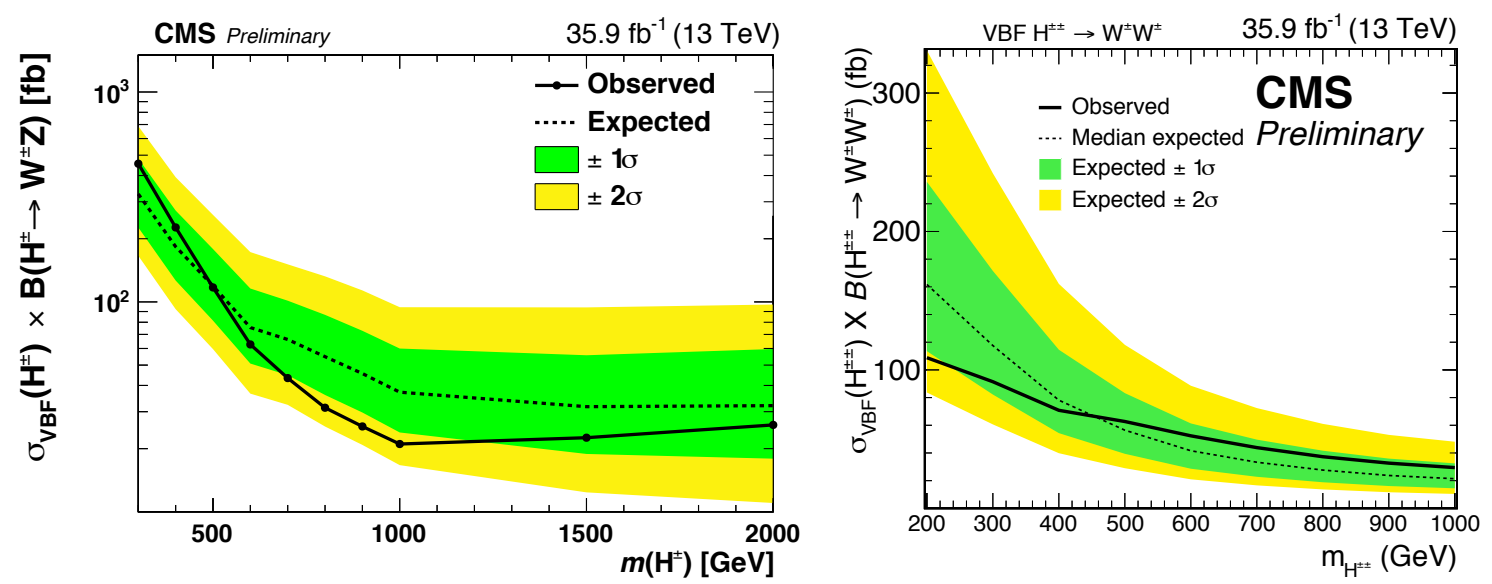

Figure 3: The observed (solid black lines) and expected (dashed lines) 95\% CL upper limits on the $\mathrm{H}^{ \pm}$ production cross section, assuming decay into $\mathrm{W}^{+} \mathrm{Z}$ (light) [12], and for the $\mathrm{H}^{++}$production cross section, assuming decay into $\mathrm{W}^{+} \mathrm{W}^{+}[13]$ (right).

\footnotetext{
${ }^{1}$ In October 2018, a new result on this channel was made public, witha larger data set of $35.9 \mathrm{fb}^{-1}$, refined analysis methods and with the $m_{\mathrm{H}^{ \pm}} \sim m_{\mathrm{t}}$ mass region included for the first time in CMS [10].
} 


\section{Summary}

An overview of the results on the charged Higgs boson searches by the CMS Collaboration is provided. The results of searches for charged Higgs bosons decaying into $c \overline{\mathrm{s}}, \mathrm{c} \overline{\mathrm{b}}, \mathrm{t} \overline{\mathrm{b}}, \tau^{+} v_{\tau}, \mathrm{W}^{+} \mathrm{Z}$ final states, and for doubly-charged Higgs bosons decaying into $\mathrm{W}^{+} \mathrm{W}^{+}$are presented, and they agree with the standard model predictions.

\section{References}

[1] G. C. Branco, P. M. Ferreira, L. Lavoura, M. N. Rebelo, M. Sher and J. P. Silva, Theory and phenomenology of two-Higgs-doublet models, Phys. Rept. 516 (2012) 1 [1106.0034].

[2] A. Djouadi, The Anatomy of electro-weak symmetry breaking. II. The Higgs bosons in the minimal supersymmetric model, Phys. Rept. 459 (2008) 1 [hep-ph/ 0503173$].$

[3] G. Senjanovic and R. N. Mohapatra, Exact Left-Right Symmetry and Spontaneous Violation of Parity, Phys. Rev. D 12 (1975) 1502.

[4] H. Georgi and M. Machacek, Doubly charged Higgs bosons, Nucl. Phys. B 262 (1985) 463.

[5] CMS Collaboration, V. Khachatryan et al., Search for a light charged Higgs boson decaying to $\mathrm{cs}$ in pp collisions at $\sqrt{s}=8 \mathrm{TeV}$, JHEP 12 (2015) 178 [1510 . 04252].

[6] CMS Collaboration, A. M. Sirunyan et al., Search for a charged Higgs boson decaying to charm and bottom quarks in proton-proton collisions at $\sqrt{s}=8 \mathrm{TeV}, 1808.06575$.

[7] CMS Collaboration, V. Khachatryan et al., Search for a charged Higgs boson in pp collisions at $\sqrt{s}=8 \mathrm{TeV}$, JHEP 11 (2015) 018 [1508.07774].

[8] CMS Collaboration, S. Chatrchyan et al., Search for a light charged Higgs boson in top quark decays in pp collisions at $\sqrt{s}=7 \mathrm{TeV}$, JHEP 07 (2012) 143 [1205. 5736].

[9] CMS Collaboration, Search for charged Higgs bosons with the $\mathrm{H}^{ \pm} \rightarrow \tau^{ \pm} v_{\tau}$ decay channel in the fully hadronic final state at $\sqrt{s}=13 \mathrm{TeV}$, CMS Physics Analysis Summary CMS-PAS-HIG-16-031, https://cds.cern.ch/record/2223865, 2016.

[10] CMS Collaboration, Search for charged Higgs bosons with the $H^{ \pm} \rightarrow \tau^{ \pm} v_{\tau}$ decay channel in proton-proton collisions at $\sqrt{s}=13 \mathrm{TeV}$, CMS Physics Analysis Summary CMS-PAS-HIG-18-014, https://cds.cern.ch/record/2640359, 2018.

[11] CMS Collaboration, A. M. Sirunyan et al., Search for charged Higgs bosons produced via vector boson fusion and decaying into a pair of $W$ and $Z$ bosons using pp collisions at $\sqrt{s}=13 \mathrm{TeV}$, Phys. Rev. Lett. 119 (2017) 141802 [1705. 02942].

[12] CMS Collaboration, Measurement of electroweak WZ production and search for new physics in pp collisions at $\sqrt{s}=13 \mathrm{TeV}$, CMS Physics Analysis Summary CMS-PAS-SMP-18-001, https://cds.cern.ch/record/2629457, 2018.

[13] CMS Collaboration, Observation of electroweak production of same-sign W boson pairs in the two jet and two same-sign lepton final state in proton-proton collisions at $13 \mathrm{TeV}$, CMS Physics Analysis Summary CMS-PAS-SMP-17-004, https://cds.cern.ch/record/2264525, 2017.

[14] CMS Collaboration, S. Chatrchyan et al., The CMS experiment at the CERN LHC, JINST 3 (2008) S08004. 